\title{
Pediatric Cerebellar Hemorrhagic Glioblastoma Multiforme
}

\author{
Peter Kalina* \\ Department of Radiology, Division of Neuroradiology, Mayo Clinic, 200 First Street, Rochester, MN 55905, USA
}

\begin{abstract}
We report the case of an 11 year old boy who presented with nausea, vomiting and ataxia. He was evaluated with computed tomography (CT) and magnetic resonance imaging (MRI). Imaging demonstrated minimal enhancement and hemorrhage of a cerebellar mass. Cerebellar glioblastoma multiforme (GBM) is extremely rare in the cerebellum at any age but especially in children. The atypical findings of minimal enhancement, cerebellar location and hemorrhagic presentation combine to make the prospective diagnosis of GBM a difficult one. This rare combination of findings has not been previously reported.
\end{abstract}

Keywords: Glioblastoma multiforme, pediatric, cerebellum.

\section{INTRODUCTION}

Glioblastoma Multiforme is a highly malignant central nervous system neoplasm. It is rarely found in the cerebellum, particularly in children. In addition, they rarely present with hemorrhage. The objective of this paper is to provide a review of the imaging features as well as to review the literature of this rare combination of findings.

\section{CASE REPORT}

An 11-year-old boy presented with a several week history of progressively increasing headaches, vomiting and motor difficulties. Physical examination was remarkable for diplopia and an ataxic gait.

Noncontrast computed tomography (CT) demonstrated a well circumscribed, hemorrhagic lesion in the midline of the posterior fossa (Fig. 1). Lateral ventricular dilatation was consistent with obstructive hydrocephalus due to fourth ventricular compression. Magnetic resonance (MR) imaging demonstrated the lesion to be essentially isointense on T1. T2 weighted sequence revealed hypointense acute hemorrhage within a well-defined hyperintense mass with minimal adjacent edema (Fig. 2). Post contrast T1 weighted images demonstrated minimal peripheral enhancement. The patient underwent emergent ventriculostomy to relieve hydrocephalus followed by suboccipital craniotomy with gross total resection of the mass. Pathologic examination was conclusive for Glioblastoma Multiforme. Metastatic evaluation was undertaken including MR of the entire spine and cerebrospinal fluid cytology. The results of these examinations were negative. Post-operative MR was negative for residual tumor. Post-operative adjuvant high-dose external beam radiation therapy directed to the posterior fossa and chemotherapy with Temozolamide was initiated.

*Address correspondence to this author at the Department of Radiology, Division of Neuroradiology, Mayo Clinic, 200 First Street, Rochester, MN 55905, USA; Tel: (507) 284-6036; Fax: (507) 255-4068; E-mail: kalina.peter@mayo.edu

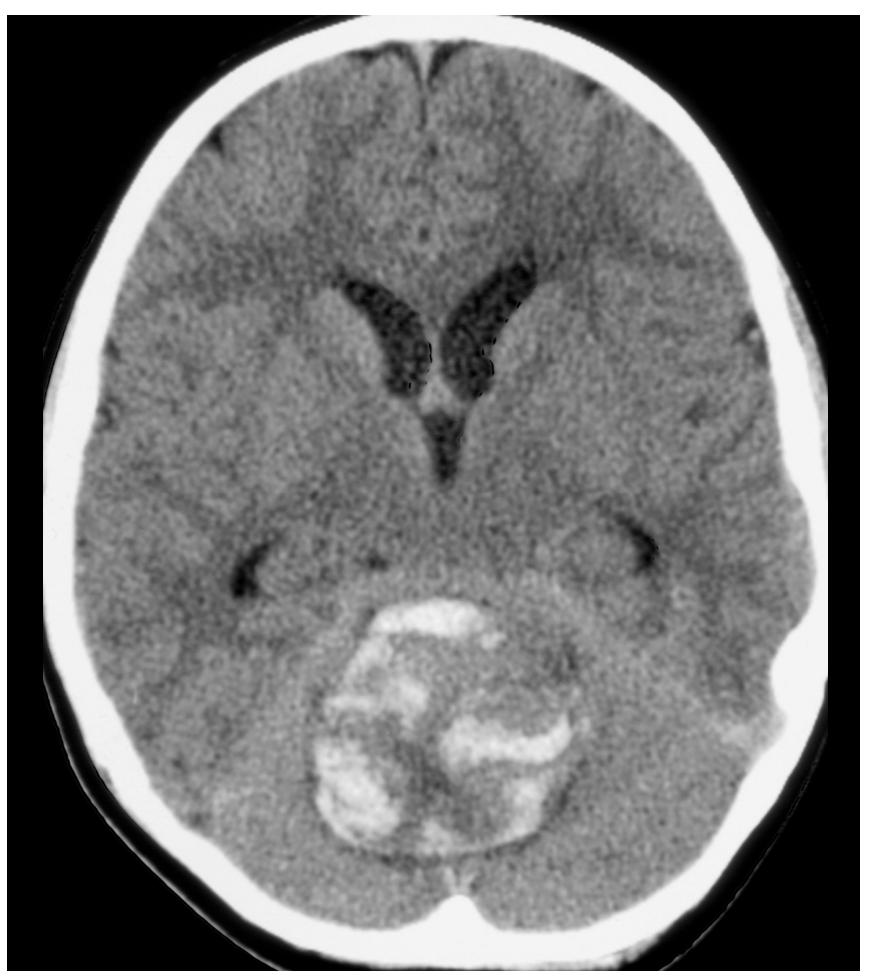

Fig. (1). Axial noncontrast CT shows a midline posterior fossa hemorrhagic mass with resultant obstructive hydrocephalus.

\section{DISCUSSION}

The clinical features of patients with cerebellar GBM are similar to those of other aggressive fast growing infratentorial tumors. Signs and symptoms include headache, nausea, vomiting, and cerebellar dysfunction including ataxia, imbalance and unsteady gait [1-4].

The imaging features of cerebellar GBM are nonspecific $[2,4-6]$. Lesions may occur laterally in the cerebellar hemispheres or in the midline within the vermis. The lesions are typically infiltrating with indistinct margins. Signal characteristics are heterogenous, often with necrotic and cystic components. A thick and irregular wall is commonly seen. 
Irregular peripheral enhancement occurs following contrast administration. Edema is usually present and obstructive hydrocephalus is common.

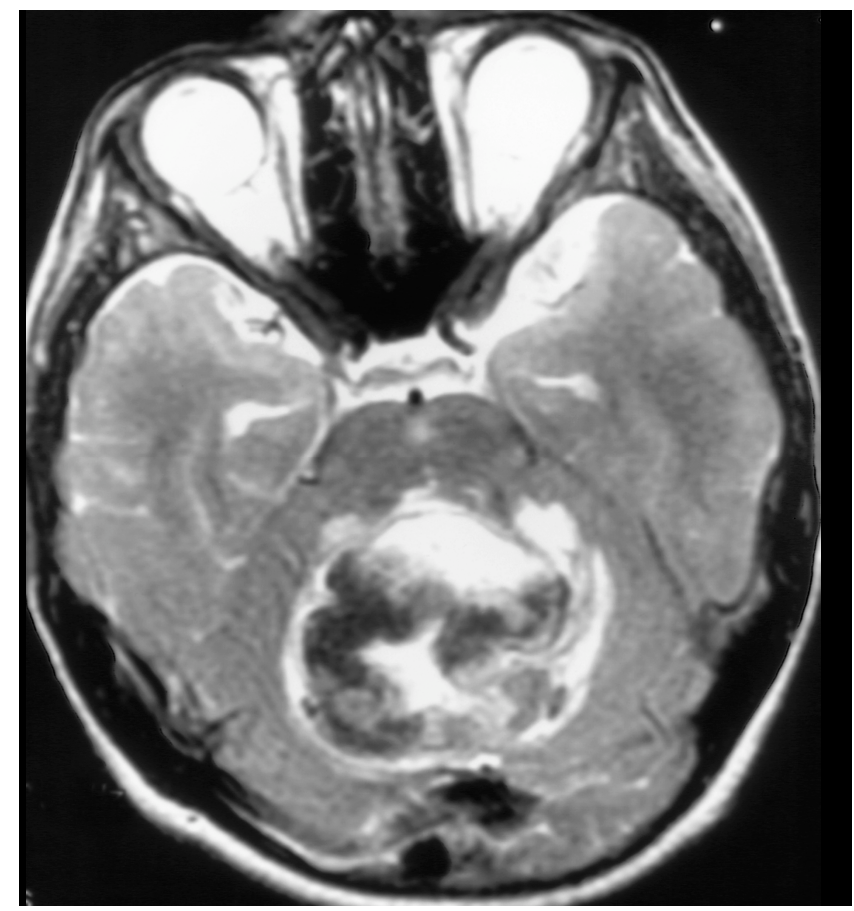

Fig. (2). Noncontrast T2 weighted MR demonstrates hypointense deoxyhemoglobin and mild surrounding edema.

The more common midline infratentorial lesions in this age group are medulloblastoma and ependymoma. They typically demonstrate more contrast enhancement. Intratumoral hemorrhage is not a common feature. The appearance of astrocytomas will vary somewhat by the histologic grade. Juvenile pilocytic astrocytoma, the most common type, is usually predominantly cystic. Tumoral hemorrhage is rare. Hemangioblastoma, while vascular, is uncommon in the pediatric population. They tend to have distinct cystic components or, solid components that enhance.

The histology and biology of cerebellar GBM is similar to that of cerebral GBM [3]. This includes malignant tumor cells, mitoses, hypercellularity, pleomorphism and neoangiogenesis. The presence of necrosis helps differentiate GBM from anaplastic astrocytoma or from well-differentiated astrocytoma $[1,4,7]$.

Brain tumors in childhood are more commonly supratentorial in the first 2-3 years of life but more commonly infratentorial from ages 4-10. The frequency is equal in children over 10 [8]. Cerebellar astrocytoma is the most common pediatric posterior fossa neoplasm [9]. It is benign and has an excellent long-term survival. The majority of pediatric cerebellar astrocytomas are of the subtype, juvenile pilocytic astrocytoma (JPA). This specific histologic type is considered grade I by World Health Organization classification. In the Kernohan's classification system [10], a well-differentiated (low-grade) astrocytoma is considered grade II. Of all cerebellar astrocytomas, $80 \%$ are Kernohan II. GBM is considered in the category of astrocytoma grade III-IV. Utilizing Burger's classification [7], lesions are graded as well differentiated astrocytoma, anaplastic astrocytoma or GBM.
GBM is rare in children in all locations. In one large series of 1,847 GBMs (all locations), less than 3\% occurred in children [11]. Cerebellar GBM is very rare at any age. Of 1,206 GBMs diagnosed over the course of 30 years, only 3 occurred in the cerebellum (all ages) [3]. However, primary infratentorial GBM is extremely rare in children. There are only 25 reported cases of pediatric primary cerebellar GBM $[1,4-6,11-14]$. At diagnosis, these children ranged in age from 2-14. There have been 8 cases of low grade cerebellar astrocytomas in children which have undergone dedifferentiation or late malignant transformation to GBM after many symptom free years post radiation therapy. Although most recurrences are seen within a few years after the initial surgery, some have recommended long-term, even life long follow-up for resected well differentiated (low-grade) cerebellar astrocytomas due to the unpredictable biological behavior [9].

As with any GBM and any pediatric malignant brain tumor, cerebellar GBM has a very poor prognosis. This is attributed to rapid tumor progression, locally aggressive behavior as well as the common findings of CSF pathway spread [5]. Early intervention including aggressive surgery as well as aggressive radiation and chemotherapy $[1,5,12$, 13] has been advocated to increase the disease free interval and to prolong survival. Despite these measures; however, survival of children with cerebellar GBM is very poor, in the range of 3-22 months [1, 4-6, 11, 14].

This case is an unusual presentation due to the presence of significant hemorrhage, well-defined margins, minimal contrast enhancement and minimal edema. There were no features helpful in making the correct specific prospective diagnosis of glioblastoma multiforme. However, GBM should be included in the differential diagnosis of a hemorrhagic infratentorial mass in a child with rapid progression of clinical findings as well as imaging findings considered atypical for the common entities that occur in the posterior fossa.

\section{CONFLICT OF INTEREST}

None declared.

\section{ACKNOWLEDGEMENT}

None declared.

\section{REFERENCES}

[1] Chin HW, Maruyama Y, Tibbs P. Cerebellar glioblastoma in childhood. J Neuro-Oncol 1984; 2: 79-84.

[2] Kuroiwa T, Numaguchi Y, Rothman MI, et al. Posterior Fossa Glioblastoma Multiforme: MR Findings. Am J Neuroradiol 1995; 16: 583-9.

[3] Luccarelli G. Glioblastoma multiforme of the cerebellum: description of three cases. Acta Neurochir 1980; 53: 107-16.

[4] Georges PM, Noterman J, Flament-Durand J. Glioblastoma of the cerebellum in children and adolescents. J Neuro-Oncol 1983; 1 275-8.

[5] Katz DS, Poe LB, Winfield JA, et al. A rare case of cerebellar glioblastoma multiforme in childhood: MR imaging. Clin Imag 1995; 19: 162-4.

[6] Rizk T, Remond J, Alhayek G. Malignant astrocytoma of the cerebellum [abstract]. Neurochirurgie 1994; 40(2): 121-6. 
[7] Burger PC, Vogel FS, Green SB, et al. Glioblastoma multiforme and anaplastic astrocytoma: pathologic criteria and prognostic implications. Cancer 1985; 56: 1106-11.

[8] Barkovich J. Pediatric Neuroimaging, Lippincott: Philadelphia 443, 2000.

[9] Schwartz AM, Ghatak NR. Malignant transformation of benign cerebellar astrocytoma. Cancer 1990; 65: 333-6.

[10] Svien HJ, Mabon RF, Kernohan JW. Astrocytomas. Proc Staff Mayo Clinic 1949; 24: 54-64.
[11] Dohrman GJ, Farwell JR, Flannery JT. Glioblastoma multiforme in children. J Neurosurg 1976; 44: 442-8.

[12] Geyer JR, Finlay JL, Boyett JM, et al. Survival of infants with malignant astrocytomas. Cancer 1995; 75: 1045-50.

[13] Wisoff JH, Boyett JM, Berger MS. Current neurosurgical management and the impact of the extent of resection in the treatment of malignant gliomas of childhood. J Neurosurg 1998; 89: 52-9.

[14] Dohrmann GJ, Dunsmore RH. Glioblastoma multiforme of the cerebellum. Surg Neurol 1975; 3:219-23.

(C) Peter Kalina; Licensee Bentham Open.

This is an open access article licensed under the terms of the Creative Commons Attribution Non-Commercial License (http://creativecommons.org/licenses/by$\mathrm{nc} / 3.0 /$ ), which permits unrestricted, non-commercial use, distribution and reproduction in any medium, provided the work is properly cited. 